\title{
Blood Group Identification Based on Fingerprint by Using 2D Discrete Wavelet and Binary Transform
}

\author{
M. Mondal ${ }^{1}$, U.K. Suma ${ }^{2}$, M. Katun ${ }^{2}$, R. Biswas ${ }^{2 *}$, Md. Rafiqul Islam ${ }^{1}$ \\ ${ }^{1}$ Mathematics Discipline, Science, Engineering and Technology School, Khulna University, Khulna 9208, Bangladesh \\ ${ }^{2}$ Department of Mathematics, Bangladesh University, Dhaka 1207, Bangladesh
}

Corresponding Author Email: rajibkumath11@gmail.com

https://doi.org/10.18280/mmc_c.802-404

Received: 1 April 2019

Accepted: 22 May 2019

\section{Keywords:}

discrete wavelets, binary transform, blood

group recognition, finger print

\begin{abstract}
Fingerprint is becoming the part of our day to day life right from our home to workplace. Now a day for security and safety purpose prime importance is given by it. Also, Fingerprint identification is one of the most popular biometric technologies and which is highly used in criminal investigations, commercial applications, and so on. The performance of a fingerprint image-matching algorithm depends heavily on the quality of the input fingerprint images. It is very important to acquire good quality images to find out gender and blood group. The use of wavelet transform improves the quality of an image and reduces noise level. In this research, different compression techniques are used to overcome this problem. Initially, different finger prints have been collected among the students of Mathematics discipline of Khulna University. Then the fingerprints have been resized to $320 * 256$ and saved in JPEG format. Then, we have used different wavelets transformation for compression of the fingerprint images. Image qualities before compression and after compression are measured by Mean Squared Error (MSE), Signalto-Noise Ratio (SNR) and Peak Signal-to-Noise Ratio (PSNR). After that the images has been pre-processing and transformed into binary images. Further, from this binary image, pixel calculation such that numbers of black and white pixels have been calculated. After that, some mathematical calculations have been done to identify the genders and blood groups. This work has been done by MATLAB programming.
\end{abstract}

\section{INTRODUCTION}

Now a day, Fingerprint is most welcome objects to our human life. In criminal investigation, fingerprint concerns a vital role because every fingerprint has an individual characteristic there was no same pattern found between two persons. Also, fingerprint pattern will remain unchanged for the life of an individual; however, the print itself may change due to permanent scars and skin diseases. Further fingerprints have general characteristic ridge patterns that allow them to be systematically identified. Fingerprint is a made of a series of ridges and valleys on the surface of the finger. Due to this characteristic of fingerprint, this is very essential to justify any criminal. Also, fingerprint is used in gender and blood group identification.

Today, fingerprint recognition has been around the longest, and there are more commercial applications of it than iris recognition. For example, different types of fingerprint recognition devices can be found for network access and physical access entry configurations. A Study of fingerprint patterns in relation to gender and blood group has been presented by Narayana et al. [1]. A study of fingerprints in relation to gender and blood group was studied by Rastogi et al. [2]. Pattern of fingerprints in different ABO blood groups was published by Bharadwaja et al. [3]. Also, finger print identification was discussed by Surinder [4]. Further, relationship between pattern of fingerprints and blood groups was submitted by Smail et al. [5]. These types of work are done by different researcher with different observation as [6-16].

This research work is to be researched the blood group identification based on fingerprint by using 2D discrete wavelet and binary transform. Initially, different fingerprints have been collected, resized and saved in JPEG format. Then from this RGB image, we have found out its binary image and its black and white pixels number. Also, the length of binary number of black pixels, number of 0's and number of 1's are calculated. All have been done by MATLAB programming. After finding these values, we have used 4 steps of calculations. Then these 4 steps of calculations, which is identifies the different blood groups of the given fingerprints.

\section{EXPERIMENTAL DATA WITH INFORMATION'S}

Table A: Experimental data with all information such as, serial number of fingerprints, name, original fingerprints, Gray scale fingerprints, Black and white fingerprints, gender and blood group has been shown in the following Table A: 


\begin{tabular}{|c|c|c|c|c|c|}
\hline Person ID & $\begin{array}{c}\text { Original } \\
\text { Fingerprints }\end{array}$ & $\begin{array}{c}\text { Gray Scale } \\
\text { Fingerprints }\end{array}$ & $\begin{array}{c}\text { Black and White } \\
\text { Fingerprints }\end{array}$ & Gender & Blood Group \\
\hline 1 & & & & Male & B+ \\
\hline 2 & & & & Male & $\mathrm{AB}^{+}$ \\
\hline 3 & & & & Female & $\mathrm{O}+$ \\
\hline 4 & & & & Male & $\mathrm{AB}+$ \\
\hline 5 & & & & Male & $\mathrm{B}^{+}$ \\
\hline 6 & & & & Male & $\mathrm{O}+$ \\
\hline 7 & & & & Male & $\mathrm{A}+$ \\
\hline 8 & & & & Female & B+ \\
\hline 9 & & & & Female & $\mathrm{O}+$ \\
\hline 10 & & & & Female & B+ \\
\hline & & & & & \\
\hline
\end{tabular}




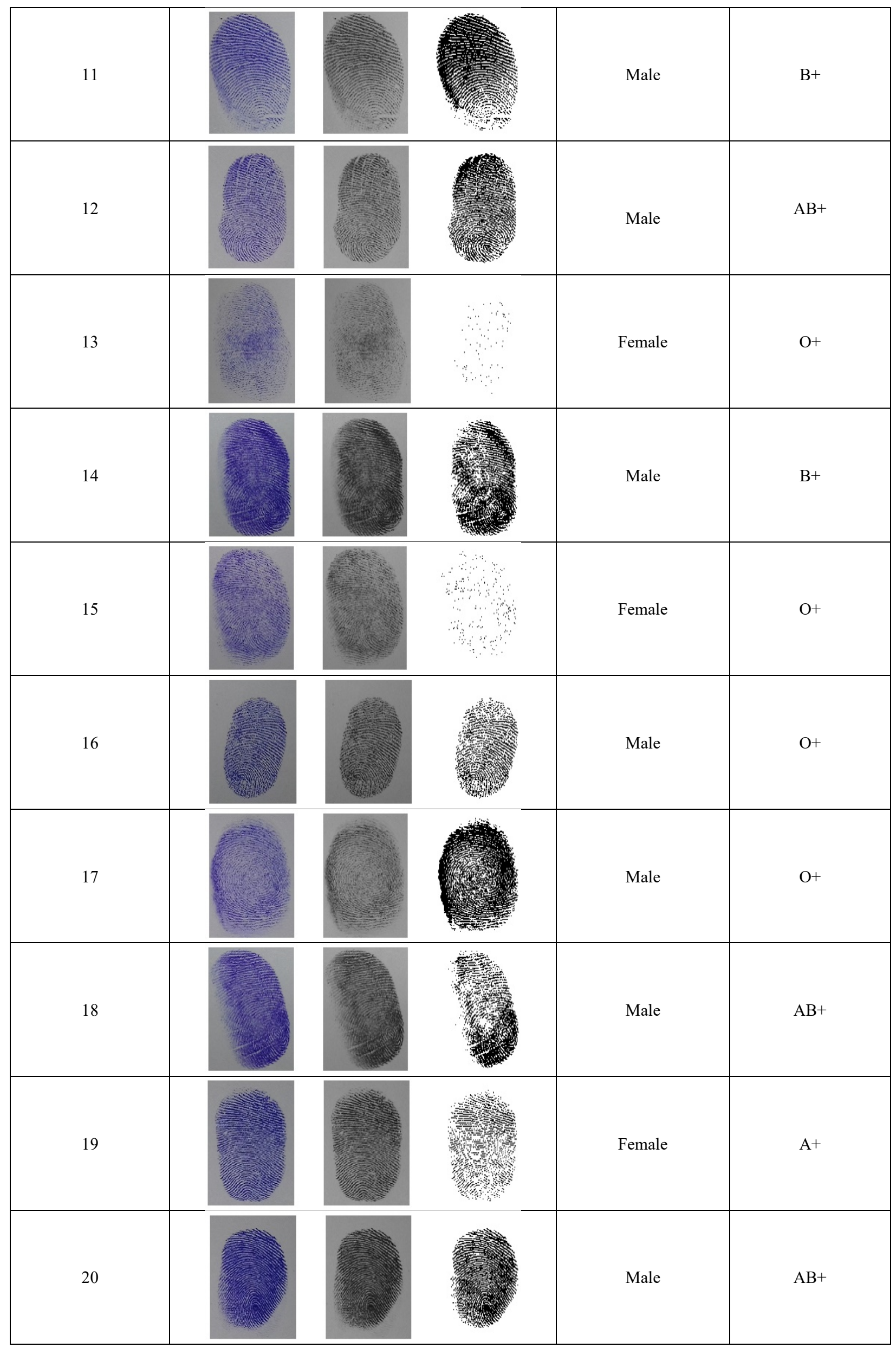




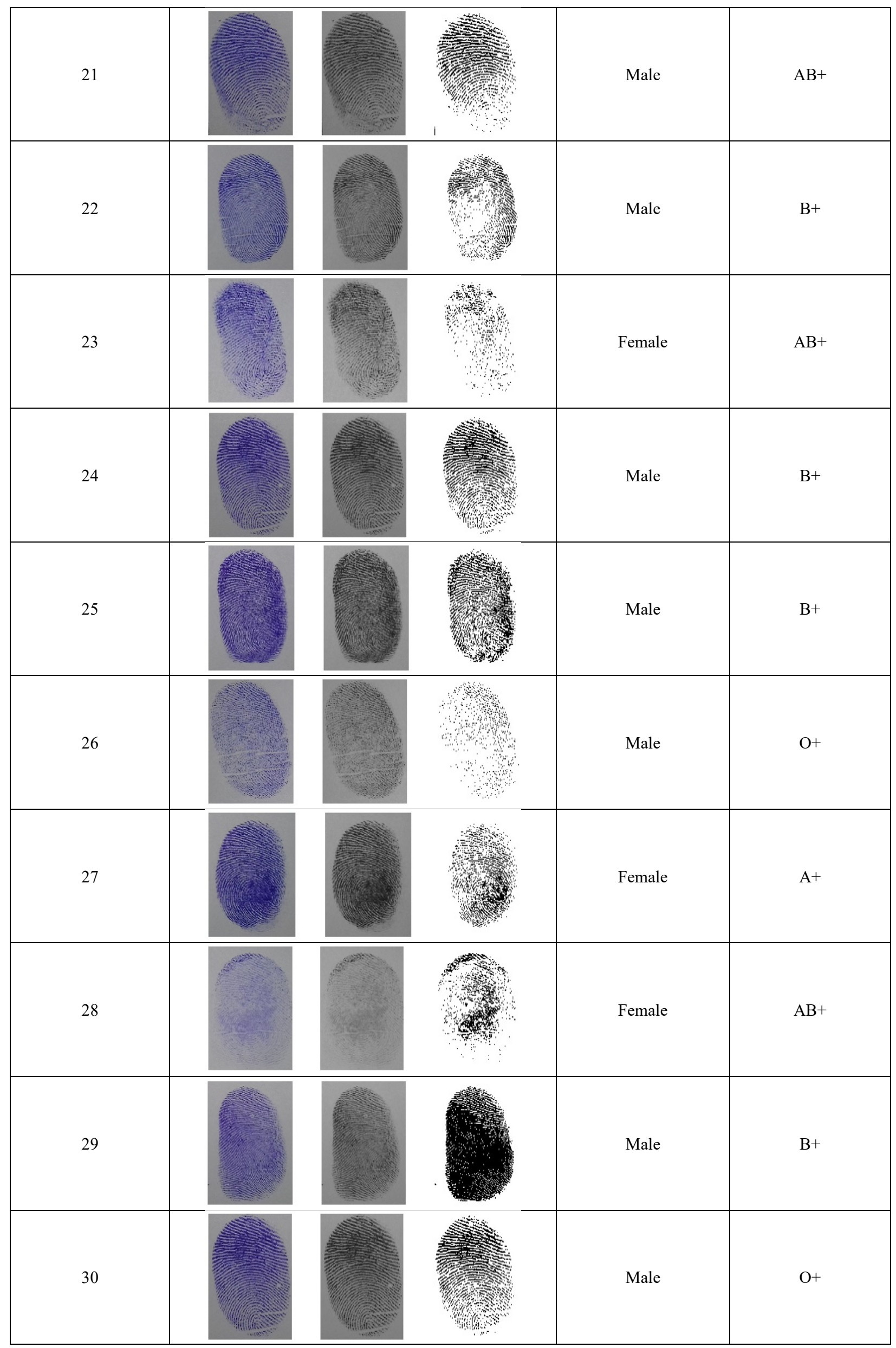




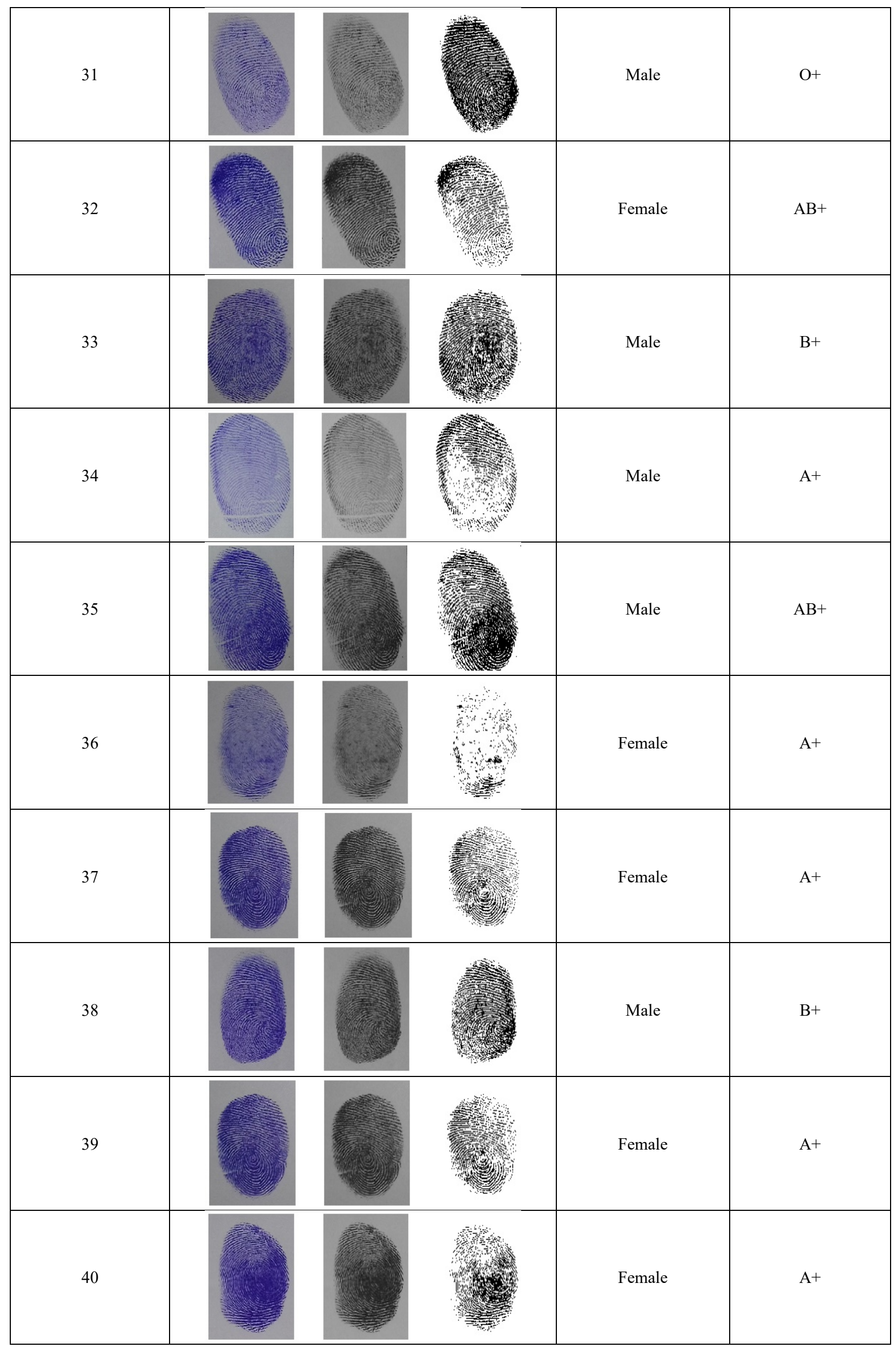




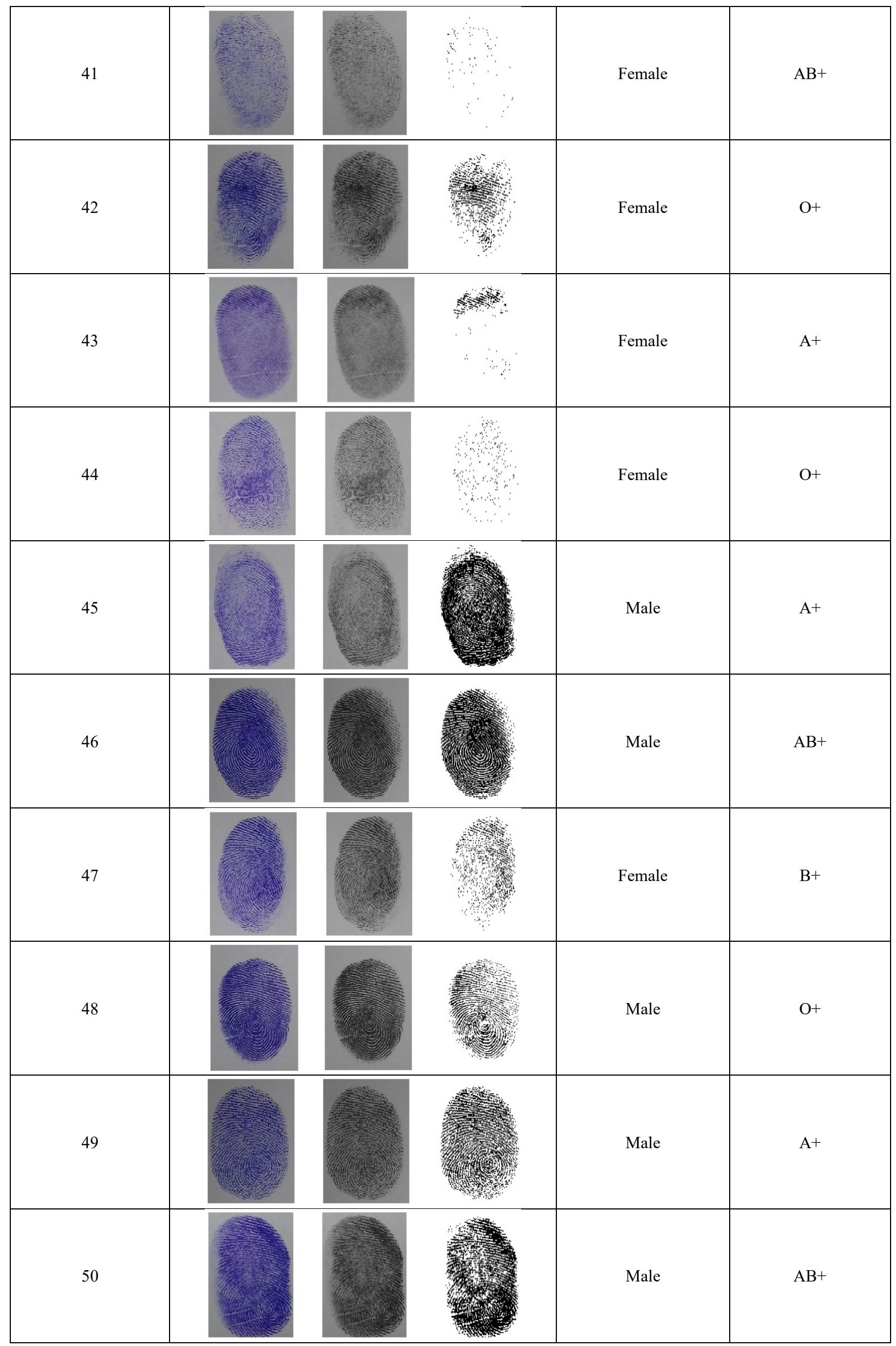




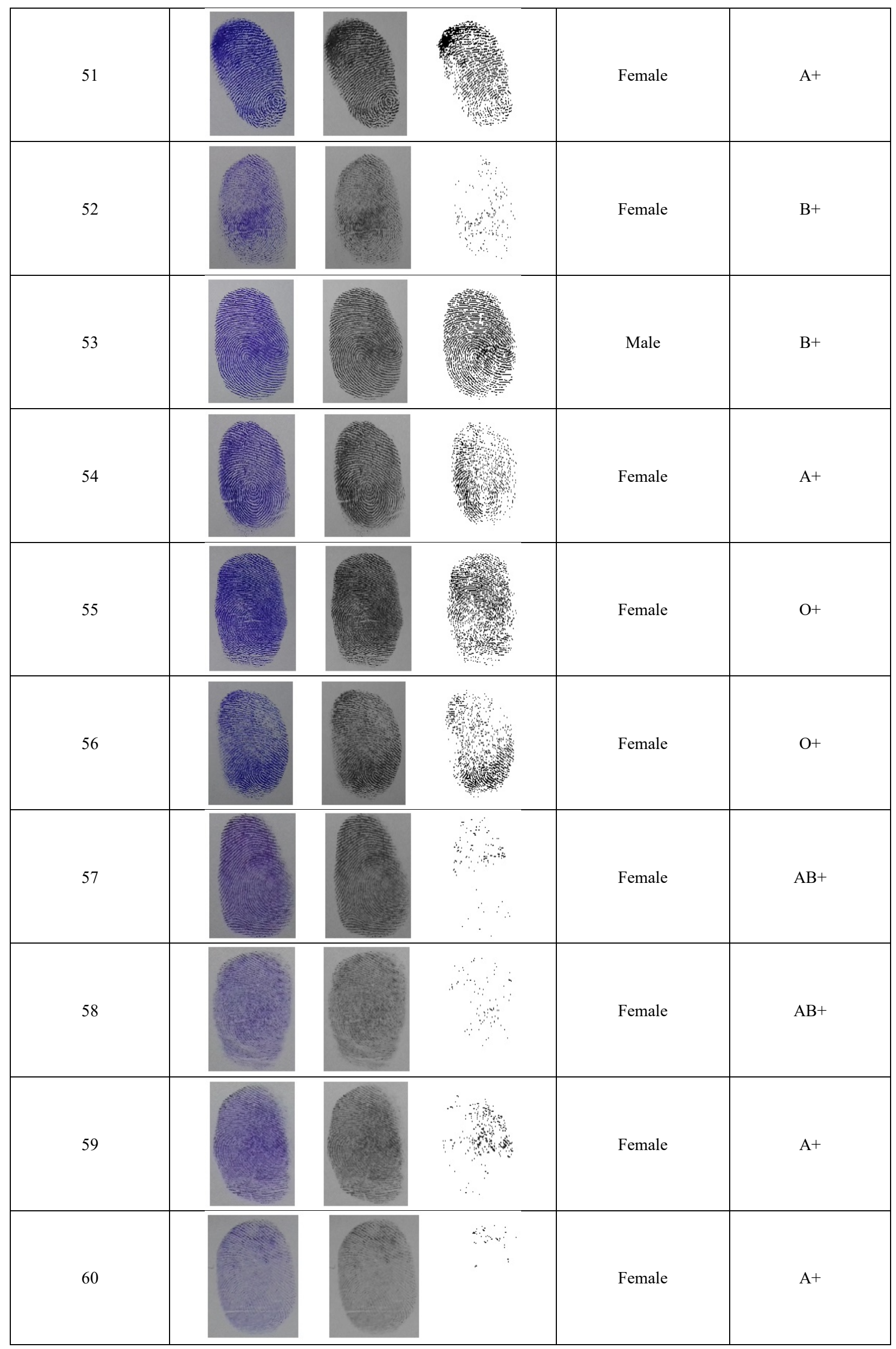




\section{WORKING PROCEDURE}

In this experiment, we have collected 60 fingerprints ( 30 male fingerprints and 30 female fingerprints) with their only $\mathrm{Rh}+\mathrm{ABO}$ blood groups. For blood group identification, we have separated the $4 \mathrm{Rh}+\mathrm{ABO}$ groups like, $\mathrm{A}+, \mathrm{B}+, \mathrm{O}+$ and $\mathrm{AB}+$. After performed these 4 steps calculations, we have obtained a good result where the following procedure has been done:

(1) At first, we have separated the all fingerprints into $4 \mathrm{Rh}+$ $\mathrm{ABO}$ groups $(\mathrm{A}+, \mathrm{B}+, \mathrm{O}+$ and $\mathrm{AB}+)$ from the obtained prepossessing fingerprints.

(2) Number of White Pixels (NWP) and Number of Black
Pixels (NBP) and Binary Length of Black Pixels (BLBP) for each fingerprint has been taken, which is already find out in chapter 3. In this chapter, we have taken those values to do perform the calculations.

(3) Subtraction (NBP-NWP) from Number of Black Pixels (NBP) to Number of White Pixels (NWP), number of 0's (total number of digits 0 ) and 1's (total number of digits 1) in the Binary Length of Black Pixels (BLBP) has been find out due to proper calculations of blood identification.

(4) All calculations have been done by MATLAB programming.

(5) The following steps has been followed for blood group identification:

\begin{tabular}{|c|c|}
\hline Step-1 & $\begin{array}{c}\text { For identification of blood group A+, } \\
\text { Calculate Quotient }=\frac{\text { NBP }}{0^{\prime} \text { sof BLBP }} \text { using MATLAB programming. Also, odd number quotient has been noted among the four blood } \\
\text { groups. }\end{array}$ \\
\hline Step-2 & $\begin{array}{c}\text { For identification of blood group B+, } \\
\text { Calculate Quotient }=\frac{\text { NWP-NBP }}{\text { B LBP }} \text { using MATLAB programming. Also, odd number quotient has been noted among the four blood } \\
\text { groups. }\end{array}$ \\
\hline Step-3 & $\begin{array}{c}\text { For identification of blood group } \mathrm{AB}+, \\
\text { Calculate Modulo }=\frac{\text { NBP }}{1 \text { 'sof BLBP }} \text { using MATLAB programming. Also, even number modulo has been noted among the four blood } \\
\text { groups. }\end{array}$ \\
\hline Step-4 & $\begin{array}{c}\text { For identification of blood group } \mathrm{O}^{+}, \\
\text {Calculate Quotient }=\frac{\mathrm{NWP}}{1 \text { 'sof BLBP }} \text { using MATLAB programming. Also, even number quotient has been noted among the four blood } \\
\text { groups. }\end{array}$ \\
\hline
\end{tabular}

\section{EXPERIMENTAL RESULTS}

Experimental results are shown in the following table and discussions have been mention in the end of the Table 1.
Here, in Table 2. Number of Even Quotient $=03$ and Number of Odd Quotient $=12$.

So, the percentage of Number of Odd Quotient $=80 \%$.

Table 1. Results for step-1 on 15 fingerprints for blood group A+

\begin{tabular}{ccccccc}
\hline Person ID & NWP & NBP & BLBP & 0's & Quotient & Observation \\
\hline 7 & 13700 & 68220 & 17 & 11 & 6201 & Odd \\
19 & 71431 & 10483 & 14 & 06 & 1747 & Odd \\
27 & 70526 & 11394 & 14 & 10 & 1139 & Odd \\
34 & 13915 & 68005 & 17 & 10 & 6800 & Even \\
36 & 77195 & 4725 & 13 & 06 & 787 & Odd \\
37 & 81077 & 843 & 10 & 04 & 211 & Odd \\
39 & 69828 & 12092 & 14 & 06 & 2015 & Odd \\
40 & 67257 & 14663 & 14 & 06 & 2443 & Odd \\
43 & 71680 & 10240 & 14 & 12 & 853 & Odd \\
45 & 38205 & 43715 & 16 & 07 & 6245 & Odd \\
49 & 22838 & 59082 & 16 & 07 & 8440 & Even \\
51 & 69865 & 12055 & 14 & 05 & 2411 & Odd \\
54 & 69590 & 12330 & 14 & 08 & 1541 & Odd \\
59 & 73640 & 8280 & 14 & 09 & 920 & Even \\
60 & 78790 & 3130 & 12 & 06 & 521 & Odd \\
\hline
\end{tabular}

Table 2. Results for step-1 on 15 fingerprints for blood group B+

\begin{tabular}{ccccccc}
\hline Person ID & NWP & NBP & BLBP & 0's & Quotient & Observation \\
\hline 1 & 6849 & 75071 & 17 & 07 & 10274 & Even \\
5 & 31095 & 50825 & 16 & 09 & 5647 & Odd \\
8 & 78270 & 3650 & 12 & 07 & 521 & Odd \\
10 & 78076 & 3844 & 12 & 07 & 549 & Odd \\
11 & 32525 & 49395 & 16 & 08 & 6174 & Even \\
14 & 28542 & 53378 & 16 & 11 & 4852 & Even \\
22 & 9327 & 72593 & 17 & 09 & 8065 & Odd \\
24 & 17486 & 64434 & 16 & 05 & 12886 & Even \\
25 & 63069 & 18851 & 14 & 06 & 3141 & Odd \\
29 & 41857 & 40063 & 16 & 05 & 8012 & Even \\
33 & 26842 & 55078 & 16 & 09 & 6119 & Odd \\
38 & 19872 & 62048 & 16 & 09 & 6894 & Even \\
47 & 29735 & 52185 & 13 & 06 & 8698 & Even \\
52 & 67893 & 14027 & 14 & 05 & 2804 & Even \\
53 & 16695 & 65225 & 16 & 05 & 13044 & Even \\
\hline
\end{tabular}


Here, Number of Even Quotient $=09$ and Number of Odd Quotient $=06$.
So, the percentage of Number of Odd Quotient $=40 \%$.

Table 3. Results for step-1 on 15 fingerprints for blood group $\mathrm{AB}+$

\begin{tabular}{ccccccc}
\hline Person ID & NWP & NBP & BLBP & 0's & Quotient & Observation \\
\hline 2 & 31004 & 50916 & 16 & 08 & 6364 & Even \\
4 & 26925 & 54995 & 16 & 06 & 9165 & Odd \\
12 & 24927 & 56993 & 16 & 07 & 8141 & Odd \\
18 & 18560 & 63360 & 16 & 08 & 7920 & Even \\
20 & 23744 & 58176 & 16 & 10 & 5817 & Odd \\
21 & 15086 & 66834 & 17 & 13 & 5141 & Odd \\
23 & 80744 & 1176 & 11 & 07 & 168 & Even \\
28 & 78256 & 3664 & 12 & 07 & 523 & Odd \\
32 & 68948 & 12972 & 14 & 07 & 1853 & Odd \\
35 & 31352 & 50568 & 16 & 10 & 5056 & Even \\
41 & 76483 & 5437 & 13 & 05 & 1087 & Odd \\
46 & 29735 & 52185 & 16 & 06 & 8698 & Even \\
50 & 32549 & 49371 & 16 & 08 & 6172 & Even \\
57 & 67596 & 14324 & 14 & 04 & 3582 & Even \\
58 & 72630 & 9290 & 14 & 06 & 1548 & Even \\
\hline
\end{tabular}

Here, Number of Even Quotient $=08$ and Number of Odd Quotient=7.
So, the percentage of Number of Odd Quotient $=46.67 \%$.

Table 4. Results for step-1 on 15 fingerprints for blood group $\mathrm{O}+$

\begin{tabular}{ccccccc}
\hline Person ID & NWP & NBP & BLBP & 0's & Quotient & Observation \\
\hline 3 & 27129 & 54791 & 16 & 08 & 6848 & Even \\
6 & 9922 & 71998 & 17 & 09 & 7999 & Even \\
9 & 67555 & 14365 & 14 & 07 & 2123 & Odd \\
13 & 71480 & 10440 & 14 & 09 & 1160 & Even \\
15 & 80744 & 1176 & 11 & 07 & 168 & Even \\
16 & 12796 & 69124 & 17 & 12 & 5760 & Even \\
17 & 36663 & 45257 & 16 & 09 & 5028 & Even \\
26 & 3676 & 78244 & 17 & 10 & 7824 & Even \\
30 & 18596 & 63324 & 16 & 05 & 12664 & Even \\
31 & 32955 & 48965 & 16 & 06 & 8160 & Even \\
42 & 79563 & 2357 & 12 & 06 & 392 & Even \\
44 & 79810 & 2010 & 11 & 05 & 402 & Even \\
48 & 31004 & 50916 & 16 & 08 & 6364 & Even \\
55 & 75560 & 6360 & 13 & 07 & 908 & Even \\
56 & 67993 & 13927 & 14 & 05 & 2785 & Odd \\
\hline
\end{tabular}

Here, Number of Even Quotient $=13$ and Number of Odd Quotient $=02$.
So, the percentage of Number of Odd Quotient $=13.33 \%$.

Table 5. Results for step-2 on 15 fingerprints for blood group A+

\begin{tabular}{ccccccc}
\hline Person ID & NWP & NBP & NWP-NBP & BLBP & Quotient & Observation \\
\hline 7 & 13693 & 68227 & 54534 & 17 & 3207 & Odd \\
19 & 71431 & 10483 & 60948 & 14 & 4353 & Odd \\
27 & 70526 & 11394 & 59132 & 14 & 4223 & Odd \\
34 & 13915 & 68005 & 54090 & 17 & 3181 & Odd \\
36 & 77195 & 4725 & 72470 & 13 & 5574 & Even \\
37 & 81077 & 843 & 80234 & 10 & 8023 & Odd \\
39 & 69828 & 12092 & 57736 & 14 & 4109 & Odd \\
40 & 67257 & 14663 & 52594 & 14 & 3756 & Even \\
43 & 71680 & 10240 & 61440 & 14 & 4338 & Even \\
45 & 38205 & 43715 & 5510 & 16 & 344 & Even \\
49 & 22838 & 59082 & 36244 & 16 & 2265 & Odd \\
51 & 69865 & 12055 & 57810 & 14 & 4129 & Odd \\
54 & 69590 & 12330 & 57260 & 14 & 4090 & Even \\
59 & 73640 & 8280 & 65360 & 14 & 4668 & Even \\
60 & 78790 & 3130 & 75660 & 12 & 6304 & Even \\
\hline
\end{tabular}

Here, Number of Even Quotient $=07$ and Number of Odd Quotient=08.
So, the percentage of Number of Odd Quotient $=53.33 \%$. 
Table 6. Results for step- 2 on 15 fingerprints for blood group B+

\begin{tabular}{ccccccc}
\hline Person ID & NWP & NBP & NWP-NBP & BLBP & Quotient & Observation \\
\hline 1 & 6849 & 75071 & 68222 & 17 & 4013 & Odd \\
5 & 31095 & 50825 & 19730 & 16 & 1233 & Odd \\
8 & 78270 & 3650 & 74620 & 12 & 6218 & Even \\
10 & 78076 & 3844 & 74232 & 12 & 6187 & Odd \\
11 & 32525 & 49395 & 16870 & 16 & 1053 & Odd \\
14 & 28542 & 53378 & 24836 & 16 & 1551 & Odd \\
22 & 9327 & 72593 & 63266 & 17 & 3721 & Odd \\
24 & 17486 & 64434 & 46948 & 16 & 2933 & Odd \\
25 & 63069 & 18851 & 44218 & 14 & 3158 & Even \\
29 & 41857 & 40063 & 1794 & 16 & 113 & Odd \\
33 & 26842 & 55078 & 28236 & 16 & 1763 & Odd \\
38 & 19872 & 62048 & 42176 & 16 & 2635 & Odd \\
47 & 29735 & 52185 & 22450 & 13 & 1725 & Odd \\
52 & 67893 & 14027 & 53866 & 14 & 3847 & Odd \\
53 & 16695 & 65225 & 48530 & 16 & 3033 & Odd \\
\hline
\end{tabular}

Here, Number of Even Quotient $=02$ and Number of Odd Quotient=13.
So, the percentage of Number of Odd Quotient $=86.66 \%$.

Table 7. Results for step-2 on 15 fingerprints for blood group $\mathrm{AB}+$

\begin{tabular}{ccccccc}
\hline Person ID & NWP & NBP & NWP-NBP & BLBP & Quotient & Observation \\
\hline 2 & 31004 & 50916 & 19912 & 16 & 1244 & Even \\
4 & 26925 & 54995 & 28070 & 16 & 1754 & Even \\
12 & 24927 & 56993 & 32066 & 16 & 2004 & Even \\
18 & 18560 & 63360 & 44800 & 16 & 2800 & Even \\
20 & 23744 & 58176 & 34432 & 16 & 2152 & Even \\
21 & 15086 & 66834 & 51748 & 17 & 3044 & Even \\
23 & 80744 & 1176 & 79568 & 11 & 7233 & Odd \\
28 & 78256 & 3664 & 74592 & 12 & 6216 & Even \\
32 & 68948 & 12972 & 55976 & 14 & 3998 & Even \\
35 & 31352 & 50568 & 19216 & 16 & 1201 & Odd \\
41 & 76483 & 5437 & 71046 & 13 & 5465 & Odd \\
46 & 29735 & 52185 & 22450 & 16 & 1403 & Odd \\
50 & 32549 & 49371 & 16822 & 16 & 1051 & Odd \\
57 & 67596 & 14324 & 53272 & 14 & 3805 & Odd \\
58 & 72630 & 9290 & 63340 & 14 & 4524 & Even \\
\hline
\end{tabular}

Here, Number of Even Quotient $=10$ and Number of Odd

So, the percentage of Number of Odd Quotient $=40 \%$. Quotient $=06$.

Table 8. Results for step-2 on 15 fingerprints for blood group $\mathrm{O}+$

\begin{tabular}{ccccccc}
\hline Person ID & NWP & NBP & NWP-NBP & BLBP & Quotient & Observation \\
\hline 3 & 27129 & 54791 & 27662 & 16 & 1778 & Even \\
6 & 9922 & 71998 & 62076 & 17 & 3651 & Odd \\
9 & 67555 & 14365 & 53190 & 14 & 3799 & Odd \\
13 & 71480 & 10440 & 61040 & 14 & 4360 & Even \\
15 & 80744 & 1176 & 79568 & 11 & 7233 & Odd \\
16 & 12796 & 69124 & 56328 & 17 & 3313 & Odd \\
17 & 36663 & 45257 & 8594 & 16 & 537 & Odd \\
26 & 3676 & 78244 & 74568 & 17 & 4336 & Even \\
30 & 18596 & 63324 & 44728 & 16 & 2795 & Odd \\
31 & 32955 & 48965 & 16010 & 16 & 1000 & Even \\
42 & 79563 & 2357 & 77206 & 12 & 6433 & Odd \\
44 & 79810 & 2010 & 77800 & 11 & 7072 & Even \\
48 & 31004 & 50916 & 19912 & 16 & 1244 & Even \\
55 & 75560 & 6360 & 69200 & 13 & 5323 & Odd \\
56 & 67993 & 13927 & 54066 & 14 & 3861 & Odd \\
\hline
\end{tabular}

Here, Number of Even Quotient $=06$ and Number of Odd Quotient $=09$.
So, the percentage of Odd Number of Quotient $=60 \%$. 
Table 9. Results for step-3 on 15 fingerprints for blood group A+

\begin{tabular}{ccccccc}
\hline Person ID & NWP & NBP & BLBP & 1's & Modulo & Observation \\
\hline 7 & 13693 & 68227 & 17 & 06 & 1 & Odd \\
19 & 71431 & 10483 & 14 & 08 & 3 & Odd \\
27 & 70526 & 11394 & 14 & 05 & 4 & Even \\
34 & 13915 & 68005 & 17 & 07 & 0 & Even \\
36 & 77195 & 4725 & 13 & 07 & 0 & Even \\
37 & 81077 & 843 & 10 & 06 & 3 & Odd \\
39 & 69828 & 12092 & 14 & 09 & 5 & Odd \\
40 & 67257 & 14663 & 14 & 08 & 7 & Odd \\
43 & 71680 & 10240 & 14 & 02 & 0 & Even \\
45 & 38205 & 43715 & 16 & 06 & 5 & Odd \\
49 & 22838 & 59082 & 16 & 09 & 6 & Even \\
51 & 69865 & 12055 & 14 & 09 & 4 & Even \\
54 & 69590 & 12330 & 14 & 05 & 0 & Even \\
59 & 73640 & 8280 & 14 & 05 & 0 & Even \\
60 & 78790 & 3130 & 12 & 06 & 4 & Even
\end{tabular}

Here, Number of Even Modulo $=09$ and Number of Odd Modulo $=06$.
So, the percentage of Number of Even Modulo $=60 \%$.

Table 10. Results for step-3 on 15 fingerprints for blood group B+

\begin{tabular}{ccccccc}
\hline Person ID & NWP & NBP & BLBP & 1's & Modulo & Observation \\
\hline 1 & 6849 & 75071 & 17 & 10 & 1 & Odd \\
5 & 31095 & 50825 & 16 & 07 & 5 & Odd \\
8 & 78270 & 3650 & 12 & 05 & 0 & Even \\
10 & 78076 & 3844 & 12 & 05 & 4 & Even \\
11 & 32525 & 49395 & 16 & 08 & 3 & Odd \\
14 & 28542 & 53378 & 16 & 05 & 3 & Odd \\
22 & 9327 & 72593 & 17 & 08 & 1 & Odd \\
24 & 17486 & 64434 & 16 & 11 & 3 & Odd \\
25 & 63069 & 18851 & 14 & 10 & 1 & Odd \\
29 & 41857 & 40063 & 16 & 11 & 1 & Odd \\
33 & 26842 & 55078 & 16 & 09 & 7 & Odd \\
38 & 19872 & 62048 & 16 & 07 & 0 & Even \\
47 & 29735 & 52185 & 13 & 07 & 0 & Even \\
52 & 67893 & 14027 & 14 & 09 & 5 & Odd \\
53 & 16695 & 65225 & 16 & 11 & 4 & Even \\
\hline
\end{tabular}

Here, Number of Even Modulo $=05$ and Number of Odd Modulo $=10$.
So, the percentage of Number of Even Modulo $=33.33 \%$.

Table 11. Results for step-3 on 15 fingerprints for blood group AB+

\begin{tabular}{ccccccc}
\hline Person ID & NWP & NBP & BLBP & 1's & Modulo & Observation \\
\hline 2 & 31004 & 50916 & 16 & 08 & 4 & Even \\
4 & 26925 & 54995 & 16 & 10 & 5 & Odd \\
12 & 24927 & 56993 & 16 & 09 & 5 & Odd \\
18 & 18560 & 63360 & 16 & 08 & 0 & Even \\
20 & 23744 & 58176 & 16 & 06 & 0 & Even \\
21 & 15086 & 66834 & 17 & 04 & 2 & Even \\
23 & 80744 & 1176 & 11 & 04 & 0 & Even \\
28 & 78256 & 3664 & 12 & 05 & 4 & Even \\
32 & 68948 & 12972 & 14 & 07 & 1 & Odd \\
35 & 31352 & 50568 & 16 & 06 & 0 & Even \\
41 & 76483 & 5437 & 13 & 08 & 5 & Odd \\
46 & 29735 & 52185 & 16 & 10 & 5 & Odd \\
50 & 32549 & 49371 & 16 & 08 & 4 & Even \\
57 & 67596 & 14324 & 14 & 10 & 6 & Even \\
58 & 72630 & 9290 & 14 & 08 & 2 & Even \\
\hline
\end{tabular}

Here, Number of Even Modulo $=10$ and Number of Odd Modulo $=05$.
So, the percentage of Number of Even Modulo $=66.67 \%$. 
Table 12. Results for step-3 on 15 fingerprints for blood group $\mathrm{O}+$

\begin{tabular}{ccccccc}
\hline Person ID & NWP & NBP & BLBP & 1's & Modulo & Observation \\
\hline 3 & 27129 & 54791 & 16 & 08 & 5 & Odd \\
6 & 9922 & 71998 & 17 & 08 & 6 & Even \\
9 & 67555 & 14365 & 14 & 07 & 1 & Odd \\
13 & 71480 & 10440 & 14 & 05 & 0 & Even \\
15 & 80744 & 1176 & 11 & 04 & 0 & Even \\
16 & 12796 & 69124 & 17 & 05 & 4 & Even \\
17 & 36663 & 45257 & 16 & 07 & 3 & Odd \\
26 & 3676 & 78244 & 17 & 07 & 5 & Odd \\
30 & 18596 & 63324 & 16 & 11 & 8 & Even \\
31 & 32955 & 48965 & 16 & 10 & 5 & Odd \\
42 & 79563 & 2357 & 12 & 06 & 5 & Odd \\
44 & 79810 & 2010 & 11 & 06 & 0 & Even \\
48 & 31004 & 50916 & 16 & 08 & 4 & Even \\
55 & 75560 & 6360 & 13 & 06 & 0 & Even \\
56 & 67993 & 13927 & 14 & 09 & 4 & Even \\
\hline
\end{tabular}

Here, Number of Even Modulo $=09$ and Number of Odd Modulo $=06$.
So, the percentage of Number of Even Modulo $=60 \%$.

Table 13. Results for step-4 on 15 fingerprints for blood group A+

\begin{tabular}{ccccccc}
\hline Person ID & NWP & NBP & BLBP & 1's & Quotient & Observation \\
\hline 7 & 13693 & 68227 & 17 & 06 & 2282 & Even \\
19 & 71431 & 10483 & 14 & 08 & 8928 & Even \\
27 & 70526 & 11394 & 14 & 05 & 14105 & Odd \\
34 & 13915 & 68005 & 17 & 07 & 1987 & Odd \\
36 & 77195 & 4725 & 13 & 07 & 11027 & Odd \\
37 & 81077 & 843 & 10 & 06 & 11582 & Even \\
39 & 69828 & 12092 & 14 & 09 & 7758 & Even \\
40 & 67257 & 14663 & 14 & 08 & 8407 & Odd \\
43 & 71680 & 10240 & 14 & 02 & 35840 & Even \\
45 & 38205 & 43715 & 16 & 06 & 6367 & Odd \\
49 & 22838 & 59082 & 16 & 09 & 2537 & Odd \\
51 & 69865 & 12055 & 14 & 09 & 7762 & Even \\
54 & 69590 & 12330 & 14 & 05 & 13918 & Even \\
59 & 73640 & 8280 & 14 & 05 & 14728 & Even \\
60 & 78790 & 3130 & 12 & 06 & 13131 & Odd \\
\hline
\end{tabular}

Here, Number of Even Quotient $=08$ and Number of Odd Quotient $=07$.
So, the percentage of Number of Even Quotient $=53.33 \%$.

Table 14. Results for step-4 on 15 fingerprints for blood group B+

\begin{tabular}{ccccccc}
\hline Person ID & NWP & NBP & BLBP & 1's & Quotient & Observation \\
\hline 1 & 6849 & 75071 & 17 & 10 & 685 & Odd \\
5 & 31095 & 50825 & 16 & 07 & 4442 & Even \\
8 & 78270 & 3650 & 12 & 05 & 15654 & Even \\
10 & 78076 & 3844 & 12 & 05 & 15615 & Odd \\
11 & 32525 & 49395 & 16 & 08 & 4065 & Odd \\
14 & 28542 & 53378 & 16 & 05 & 5708 & Even \\
22 & 9327 & 72593 & 17 & 08 & 1165 & Odd \\
24 & 17486 & 64434 & 16 & 11 & 1589 & Odd \\
25 & 63069 & 18851 & 14 & 10 & 6306 & Even \\
29 & 41857 & 40063 & 16 & 11 & 3805 & Odd \\
33 & 26842 & 55078 & 16 & 09 & 2982 & Even \\
38 & 19872 & 62048 & 16 & 07 & 2838 & Even \\
47 & 29735 & 52185 & 13 & 07 & 4247 & Odd \\
52 & 67893 & 14027 & 14 & 09 & 7543 & Odd \\
53 & 16695 & 65225 & 16 & 11 & 1517 & Odd \\
\hline
\end{tabular}

Here, Number of Even Quotient $=06$ and Number of Odd Quotient $=09$.
So, the percentage of Number of Even Quotient $=40 \%$. 
Table 15. Results for step-4 on 15 fingerprints for blood group AB+

\begin{tabular}{ccccccc}
\hline Person ID & NWP & NBP & BLBP & 1's & Quotient & Observation \\
\hline 2 & 31004 & 50916 & 16 & 08 & 4250 & Even \\
4 & 26925 & 54995 & 16 & 10 & 2692 & Even \\
12 & 24927 & 56993 & 16 & 09 & 2769 & Odd \\
18 & 18560 & 63360 & 16 & 08 & 2320 & Even \\
20 & 23744 & 58176 & 16 & 06 & 3957 & Odd \\
21 & 15086 & 66834 & 17 & 04 & 3771 & Odd \\
23 & 80744 & 1176 & 11 & 04 & 20186 & Even \\
28 & 78256 & 3664 & 12 & 05 & 15651 & Odd \\
32 & 68948 & 12972 & 14 & 07 & 9849 & Odd \\
35 & 31352 & 50568 & 16 & 06 & 5225 & Odd \\
41 & 76483 & 5437 & 13 & 08 & 9560 & Even \\
46 & 29735 & 52185 & 16 & 10 & 2973 & Odd \\
50. & 32549 & 49371 & 16 & 08 & 4068 & Even \\
57 & 68570 & 13350 & 14 & 10 & 6857 & Odd \\
58 & 72630 & 9290 & 14 & 08 & 9078 & Even \\
\hline
\end{tabular}

Here, Number of Even Quotient $=07$ and Number of Odd Quotient $=08$.
So, the percentage of Number of Even Quotient $=46.67 \%$.

Table 16. Results for step-4 on 15 fingerprints for blood group $\mathrm{O}+$

\begin{tabular}{ccccccc}
\hline Person ID & NWP & NBP & BLBP & 1's & Quotient & Observation \\
\hline 3 & 27129 & 54791 & 16 & 08 & 3392 & Even \\
6 & 9922 & 71998 & 17 & 08 & 1240 & Even \\
9 & 67555 & 14365 & 14 & 07 & 9650 & Even \\
13 & 71480 & 10440 & 14 & 05 & 14296 & Even \\
15 & 80744 & 1176 & 11 & 04 & 20176 & Even \\
16 & 12796 & 69124 & 17 & 05 & 2569 & Odd \\
17 & 36663 & 45257 & 16 & 07 & 5238 & Even \\
26 & 3676 & 78244 & 17 & 07 & 525 & Odd \\
30 & 18596 & 63324 & 16 & 11 & 1690 & Even \\
31 & 32955 & 48965 & 16 & 10 & 5495 & Odd \\
42 & 79563 & 2357 & 12 & 06 & 13302 & Even \\
44 & 79810 & 2010 & 11 & 06 & 13260 & Even \\
48 & 31004 & 50916 & 16 & 08 & 3876 & Even \\
55 & 75560 & 6360 & 13 & 06 & 12592 & Even \\
56 & 67993 & 13927 & 14 & 09 & 7554 & Even \\
\hline
\end{tabular}

Here, Number of Even Quotient $=12$ and Number of Odd Quotient $=03$.

So, the percentage of Number of Even Quotient $=80 \%$.

\section{RESULTS AND DISCUSSION}

From the above Table-1, Table-2, Table- 3 and Table- 4 , we can see that the percentage of Odd Number Quotient for blood group $(\mathrm{A}+)$ is greater than others blood groups. So, we conclude that, when we apply Step-1 for any one fingerprint whose blood group is unknown, his/her blood group probability $(\mathrm{A}+)$ will be $80 \%$.

Also, we can see that the percentage of Odd Number Quotient for blood group $(\mathrm{B}+)$ is greater than others blood groups. So, we conclude that, when we apply Step-2 for any one fingerprint whose blood group is unknown, his/her blood group probability $(\mathrm{B}+)$ will be $86.67 \%$. This observation is shown in the Table-5 to Table- 8 .

Further, the percentage of Even Number of Modulo for blood group $(\mathrm{AB}+)$ is greater than others blood groups. So, we conclude that, when we apply Step-3 for any one fingerprint whose blood group is unknown, his/her blood group probability $(\mathrm{AB}+)$ will be $66.67 \%$ all have are presented in the Table-9 to Table-12.

Again, from the above Table-13 toTable-16, we can see that the percentage Number of Even Quotient for blood group O+ is greater than others blood groups. So, we conclude that, when we apply Step-4 for any one fingerprint whose blood group is unknown, his/her $\mathrm{O}+$ blood group probability will be $80 \%$.

Therefore we conclude that, when we apply Step-1 for any one fingerprint whose blood group is unknown, his/her A+ blood group probability will be $80 \%$; for Step-2 probability of $\mathrm{B}+$ blood group will be $80.67 \%$; for Step-3 probability of $\mathrm{AB}+$ blood group will be $66.67 \%$ and for Step-4 probability of $\mathrm{O}+$ blood group will be $80 \%$ which are shown in the above Table1 to Table-16.

\section{REFERENCES}

[1] Narayana, B.L., Rangaiah, Y.K.C., Khalid, M.A. (2016). Study of fingerprint patterns in relation to gender and blood group. J Evolution Med. Dent. Society, 5(14): 530533. https://doi.org/10.14260/jemds/2016/144

[2] Rastogi, D.P., Ms Pillai, K.R. (2010). A study of fingerprints in relation to gender and blood group. J Indian Acad Forensic Med, 32(1): 11-14.

[3] Bharadwaja, A., Saraswat, P.K., Agrawal, S.K. (2004). Pattern of fingerprints in different ABO blood groups. Journal of Forensic Medicine and Toxicology, 21(2): 4952 . 
[4] Surinder, N. (1984). Finger Print Identification. Gita Press, 1-15. https://doi.org/10.1016/S01406736(00)51333-6

[5] Smail, H.O., Wahab, D.A., Abdullah, Z.Y. (2019). Relationship between pattern of fingerprints and blood groups. Journal of Advanced Laboratory Research in Biology, 10: 84-90.

[6] Agrawal, H., Choubey, S. (2014). Fingerprint based gender classification using multi-class SVM. International Journal of Advanced Research in Computer Engineering \& Technology, 3(8): 2575-2580.

[7] Arulkumaran, T., Sankaranarayanan, D.P.E., Sundari, D.G. (2013). Fingerprint Based Age Estimation Using 2D Discrete Wavelet Transforms and Principal Component Analysis. International Journal of Advanced Research in Electrical, Electronics and Instrumentation Engineering, 2(3): 1060-1066.

[8] Balaji, S., Venkatram, N. (2008). Filtering of noise in fingerprint images. International Journal of Systems and Technologies, 1: 87-94.

[9] Bhavana, D., Ruchi, J., Prakash, T. (2013). Study of fingerprint patterns in relationship with blood group and gender- a statistical review. Res J Forensic Society, 1(1): 15-17.

[10] Borra, S.R., Reddy, G.J., Reddy, S. (2014). Fingerprint image de-noising using wave atom transform. International Journal of Computer Science Engineering and Information Technology Research, 4: 69-76.
[11] Chen, G.Y., Kegl, B. (2007). Image denoising with complex ridgelets. Pattern Recognition, 40: 578-585.

[12] Devnath, L., Islam, R. (2016). Fingerprint image denoising by various filters for different noise using wavelet transform. American International Journal of Research in Science, Technology, Engineering \& Mathematics, 13(1): 39-44. https://doi.org/10.13140/RG.2.1.2448.3448

[13] Ganesh, B., Dongre, D.S., Jagade, M. (2015). A review and study on fingerprint based gender classification using classified techniques. International Journal of Scientific Research, 6: 596-599.

[14] Gopinathan, S.K., Thangavel, P.R. (2015). Wavelet and FFT based image de-noising using non-linear filters. International Journal of Electrical and Computer Engineering, $\quad 5$ : 1018-1026. https://doi.org/10.11591/ijece.v5i5.pp1018-1026

[15] Gornale, S.S., Hangarge, M., Pardeshi, R., Kruthi, R. (2015). Haralick feature descriptors for gender classification using fingerprints: A machine learning approach. International Journal of Advanced Research in Computer Science and Software Engineering, 5: 72-78.

[16] Gupta, S., Rao, A.P. (2014). Fingerprint based gender classification using discrete wavelet transform \& artificial neural network. International Journal of Computer Science and Mobile Computing, 3(4): 12891296. https://doi.org/10.13140/RG.2.2.15292.69769 